\title{
Self-assessment questions: Making sense of gut-brain signals
}

\author{
Graham J Dockray
}

1 Ingestion of lipid:

(a) Releases the intestinal hormone cholecystokinin (CCK)

(b) Decreases the blood oxygen level-dependent (BOLD) signal in magnetic resonance images of hypothalamus

(c) Increases central nervous system (CNS) responses to visual and auditory cues for sadness

(d) Acutely increases vagal afferent neuron discharge

(e) Inhibits food intake via ghrelin release

2 Vagal afferent neurons:
(a) Mediate painful sensations from the stomach
(b) Exhibit leptin resistance in obesity
(c) Are inhibited by cholecystokinin (CCK)
(d) Constitute the afferent arm of reflexes leading to inhibi- tion of gastric emptying
(e) Exhibit nutrient-dependent neurochemical plasticity

3 The gut microbiota:

(a) Change in obesity

(b) Might decrease food intake via release of cholecystokinin (CCK)

(c) Contribute to obesity through increased energy extraction

(d) Do not influence vagal afferent responses to leptin

(e) Participate in gut-brain but not brain-gut signalling

Answers to these self-assessment questions can be found on page 592 . 